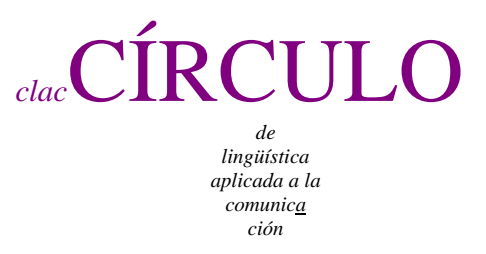

$63 / 2015$

\title{
HIÉRARCHIE DES RELATIONS SÉMANTIQUES INTERPRÉDICATIONNELLES ET APPROPRIATION EN FRANÇAIS L2
}

\author{
Manon Buysse \\ Vrije Universiteit Brussel \\ manon buysse at vub ac be
}

Résumé

Le développement de la jonction interpropositionnelle en français L2 constitue un objet de recherche important en linguistique acquisitionnelle. De nombreuses études se sont centrées sur le développement de structures complexes spécifiques, souvent sur la base du rapport sémantique entre les événements qu’elles expriment. Il s’agit entre autres des complétives (Diessel \& Tomasello 2001), des interrogatives indirectes (Eckman et al. 1989), des temporelles (Noyau \& Vasseur 1986, Degand \& Hadermann 2009) ou des causales (Benazzo 2004).

Notre contribution examine l'émergence des différentes relations sémantiques entre événements ou états, exprimables au moyen de la jonction interpropositionnelle et interprédicationnelle. Nous voulons montrer comment le répertoire de liens sémantiques exprimés évolue dans l'acquisition d'une langue seconde selon l'âge et le niveau de compétence des apprenants. A cette fin, nous analysons des narrations orales semi-

Manon Buysse. 2015.

Hiérarchie des relations sémantiques interprédicationnelles et appropriation en français L2 Círculo de Lingüística Aplicada a la Comunicación 63, 97-121.

http://www.ucm.es/info/circulo/no63/buysse.pdf

http://revistas.ucm.es/index.php/CLAC

http://dx.doi.org/10.5209/rev_CLAC.2015.v63.50171

(C) 2015 Manon Buysse

Círculo de Lingüística Aplicada a la Comunicación (clac)

Universidad Complutense de Madrid. ISSN 1576-4737. http://www.ucm.es/info/circulo 
spontanées produites par des élèves de l'enseignement secondaire en Flandre, qui apprennent le français comme langue seconde. Notre hypothèse de recherche sera basée sur la hiérarchie des relations interprédicationnelles sémantiques proposée par la Grammaire du Rôle et de la Référence (Role and Reference Grammar ; Van Valin \& LaPolla 1997, Van Valin 2005).

Cette contribution se concentrera sur plusieurs questions de recherche liées aussi bien à la nature sémantique des jonctions qu’à leur réalisation syntaxique: les apprenants acquièrent-ils en premier lieu les relations interprédicationnelles sémantiquement fortes, inclues les catégories structurellement complexes et peut-être plus difficiles à conceptualiser? Les premiers types de liens à être productifs diffèrent-ils selon le niveau syntaxique de la jonction? La production des apprenants du français L2 est-elle semblable à celle de locuteurs natifs de la même langue ?

Mots clé : acquisition langue seconde, sémantique, jonction interprédicationnelle, séquences développementales

\section{Abstract}

The hierarchy of interpredicational semantic relations and second language acquisition The development of clause linkage in French as a second language has been an important research topic in the field of language acquisition. Several studies have focused on the development of specific complex structures, often based on the semantic relationship they establish between multiple events : complement clauses (Diessel \& Tomasello 2001), indirect interrogatives (Eckman et al. 1989), temporal (Noyau \& Vasseur 1986, Degand \& Hadermann 2009) or causative subclauses (Benazzo 2004).

Our contribution examines the emergence of different semantic relations between events or states, expressed by means of interclausal and interpredicational linkage. We would like to show how learners' repertoire of productive semantic links evolves according to their age and level of competence while acquiring a second language. For this purpose, we have analyzed semi-spontaneous oral narratives produced by secondary school pupils in Flanders, learning French as a second language. Our research hypothesis will be based on the hierarchy of semantic interpredicational relations proposed within the framework of Role and Reference Grammar (Van Valin \& LaPolla 1997, Van Valin 2005). 
This contribution focuses on several research questions related at once to the semantic nature of clause links and to their syntactic réalisation : do learners acquire semantically strong interpredicational relations before all others, and does this apply as well to those categories which are structurally complex and therefore perhaps more difficult to conceptualise ? Are different types of links more productive or produced earlier in development at different syntactic levels of juncture ? Do L2 learners' narratives in French resemble those of native speakers of the same language?

Keywords: second language acquisition, semantics, clause linkage, developmental sequences

Table des matières

1. Introduction, 99

2. Cadre théorique : la Grammaire du Rôle et de la Référence (RRG), 100

3. Méthodologie et questions de recherche, 104

4. Données observées, 107

5. Discussion des hypothèses formulées, 114

6. Conclusions, 119

Bibliographie, 120

\section{Introduction}

Le développement de la jonction interpropositionnelle en français L2 constitue un objet de recherche important en linguistique acquisitionnelle. De nombreuses études se sont centrées sur le développement de structures complexes spécifiques, souvent sur la base du rapport sémantique entre les événements qu’elles expriment. Il s’agit entre autres des complétives (Diessel \& Tomasello 2001), des interrogatives indirectes (Eckman et al. 1989), des temporelles (Noyau \& Vasseur 1986, Degand \& Hadermann 2009) ou des causales (Benazzo 2004). 
Notre contribution examine l'émergence des différentes relations sémantiques entre événements ou états, exprimables au moyen de la jonction interpropositionnelle et interprédicationnelle. Nous voulons montrer comment le répertoire de liens sémantiques exprimés évolue dans l'acquisition d'une langue seconde selon l'âge et le niveau de compétence des apprenants. A cette fin, nous analysons des narrations orales semispontanées produites par des élèves de l'enseignement secondaire en Flandre, qui apprennent le français comme langue seconde. Notre hypothèse de recherche sera basée sur la hiérarchie des relations interprédicationnelles sémantiques proposée par la Grammaire du Rôle et de la Référence (Role and Reference Grammar ; Van Valin \& LaPolla 1997, Van Valin 2005).

2. Cadre théorique : la Grammaire du Rôle et de la Référence (RRG)

La Grammaire du Rôle et de la Référence prétend proposer un modèle fonctionnel universel permettant de rendre compte de l'interaction entre les composantes syntaxique, sémantique et pragmatique d'une langue donnée. A côté d'une structure hiérarchisée de la proposition (layered structure of the clause), qui distingue trois plans au niveaux desquels peut être instaurée une relation syntaxique entre unités, elle propose aussi un continuum sémantique, sur lequel seront positionnées les jonctions interprédicationnelles sur la base de l’intensité du lien sémantique entre les prédications: la Hiérarchie des relations sémantiques interprédicationnelles (Interclausal semantic relations hierarchy) ou HRSmI. Enfin, la RRG relie ces deux plans dans un troisième schème général, qui réunit les continuums syntaxique et sémantique: la Hiérarchie des relations interprédicationnelles (Interclausal relations hierarchy). Selon la RRG, les liens syntaxiquement les plus forts correspondront généralement aux liens sémantiques les plus étroits, et la même équivalence entre les deux plans se retrouvera au niveau des liens faibles (cf. Van Valin \& LaPolla 1997, Van Valin 2005).

Dans le tableau 1, nous fournissons simplement un aperçu des relations syntaxiques pertinentes pour la langue française, en y ajoutant chaque fois un exemple. Les relations en question sont organisées selon la Hiérarchie des relations syntaxiques interprédicationnelles (Interclausal syntactic relations hierarchy) ou HRSxI, c'est-àdire que les types de liens interprédicationnels plus proches du pôle supérieur ou 'fort' 
sont censés être syntaxiquement plus étroits, tandis que les liens plus proches du pôle inférieur sont considérés comme syntaxiquement plus faibles.

Tableau 1: Types de jonction et de nexus en français

\begin{tabular}{|l|l|l|}
\hline Jonction & Type de nexus & Exemple \\
\hline Nucléaire & Cosubordination & Le chien a fait tomber le bocal. \\
\hline Cellulaire & Cosubordination & Je veux bien t’aider. \\
\hline & $\begin{array}{l}\text { Subord. } \\
\text { 'complément' }\end{array}$ & Fumer est mauvais pour la santé. \\
\hline & Subord. 'ad(verbiale)’ & Tu m’appelles quand tu es prête? \\
\hline & Coordination & Le professeur a dit à Lucien de se taire. \\
\hline & $\begin{array}{l}\text { Cosubordination } \\
\text { 'complément' }\end{array}$ & Luc n'aime pas voyager et moi non plus. \\
\hline & Subord. 'ad(verbiale)’ & Je suis fatigué parce que je suis malade. \\
\hline & Coordination & $\begin{array}{l}\text { J’aime mes parents, mais je ne les vois pas } \\
\text { souvent. }\end{array}$ \\
\hline
\end{tabular}

Pour plus de précisions concernant la structure hiérarchisée de la proposition, les types de relations syntaxiques possibles, et l'interaction entre la syntaxe et la sémantique selon la RRG, nous référons à Van Valin \& LaPolla 1997, Van Valin 2005 ou Buysse 2014.

Nous nous centrons à présent sur l'émergence des liens sémantiques interprédicationnels dans l'appropriation d'une L2 à travers l'observation de plusieurs stades d'acquisition du français langue seconde.

La Hiérarchie des relations sémantiques interprédicationnelles (HRSmI) distingue vingt combinaisons différentes pour rendre compte des relations sémantiques engendrées entre prédications sur divers plans syntaxiques (Van Valin 2005). Elles sont répertoriées selon la puissance du lien entre les événements ou les actions reliés au moyen de la jonction interprédicationnelle. Les types de relations au sommet de la hiérarchie réunissent deux entités exprimant plusieurs aspects d'un seul événement ou situation, tandis que les types de relations au bas de la hiérarchie lient deux événements 
autonomes mais interagissant d'une certaine manière. Le tableau 2 donne un aperçu de la hiérarchie des rapports sémantiques à partir du pôle d'intensité maximal jusqu'au pôle d'intensité minimal. Chaque type de rapport sera illustré par un exemple. L’appellation de certaines catégories sera explicitée par après.

Tableau 2: Types de jonction sémantique selon la RRG

\begin{tabular}{|c|c|}
\hline Type sémantique & Exemples \\
\hline Causatif [1] & $\begin{array}{l}\text { Julie a peint la table rouge. } \\
\text { Je ferai manger les gâteaux à Jean. }\end{array}$ \\
\hline Aspectuel & $\begin{array}{l}\text { Christiane commençait à pleurer. } \\
\text { Frédéric continuait à chanter. }\end{array}$ \\
\hline Sous-événement modifiant & $\begin{array}{l}\text { Manière: Marie est entrée en chantant. } \\
\text { Position: Elle était assise lisant un livre. } \\
\text { Moyen: Thomas a ouvert le paquet en le coupant avec } \\
\text { un couteau. }\end{array}$ \\
\hline Action psychologique & $\begin{array}{l}\text { Sophie a décidé de partir. } \\
\text { Louis veut aller au cinéma. }\end{array}$ \\
\hline But & $\begin{array}{l}\text { Gérard est allé au supermarché pour acheter du lait. } \\
\text { Suzanne a fermé la fenêtre pour que son fils puisse } \\
\text { dormir. }\end{array}$ \\
\hline Jussif & $\begin{array}{l}\text { Patrice a demandé à l'étudiant de partir. } \\
\text { Le roi a ordonné aux troupes d'attaquer la ville. }\end{array}$ \\
\hline Causatif [2] & $\begin{array}{l}\text { Léon a contraint Anne à participer à la fête. } \\
\text { Je laisserai Jean manger les gâteaux. }\end{array}$ \\
\hline Perception directe & $\begin{array}{l}\text { Lucie a vu l’enfant ouvrir la porte. } \\
\text { Béatrice a entendu les visiteurs arriver. }\end{array}$ \\
\hline Perception indirecte & $\begin{array}{l}\text { (en regardant un bureau vide) Je vois que Marc est allé à } \\
\text { la maison tôt aujourd'hui. }\end{array}$ \\
\hline Attitude propositionnelle & $\begin{array}{l}\text { Cédric croit que les extraterrestres existent. } \\
\text { Les fans veulent que leur équipe gagne le match. }\end{array}$ \\
\hline Cognition & $\begin{array}{l}\text { Joseph sait que la terre est ronde. } \\
\text { Josiane pense au fait que George l'a refusée. }\end{array}$ \\
\hline
\end{tabular}




\begin{tabular}{|c|c|}
\hline Discours indirect & $\begin{array}{l}\text { Alice criait qu'elle avait oublié son manteau dans le } \\
\text { train. } \\
\text { François disait que ses amis étaient corrompus. }\end{array}$ \\
\hline Discours direct & $\begin{array}{l}\text { Alice criait: “J’ai oublié mon manteau dans le train!” } \\
\text { François disait: “Mes amis sont corrompus.” }\end{array}$ \\
\hline $\begin{array}{l}\text { Circonstances } \\
\text { temporelles }\end{array}$ & $\begin{array}{l}\text { Robert a perdu ses gants là où il s’était assis. } \\
\text { J'ai brossé mes dents avant de quitter la maison. }\end{array}$ \\
\hline Cause & $\begin{array}{l}\text { L’enfant pleurait parce qu’il était tombé. } \\
\text { Comme tout le monde est là, la réunion commence. }\end{array}$ \\
\hline Condition & $\begin{array}{l}\text { S’il pleut, nous ne pourrons pas aller nager. } \\
\text { N'oublie pas ton parapluie, au cas où il pleuve. }\end{array}$ \\
\hline Concession & $\begin{array}{l}\text { Guillaume est arrivé au boulot à temps, même s'il } \\
\text { neigeait. } \\
\text { J'aime ma soeur bien qu'elle soit parfois impossible. }\end{array}$ \\
\hline Situations simultanées & $\begin{array}{l}\text { Didier dansait, et en même temps Gracienne jouait du } \\
\text { piano. } \\
\text { Michelle nettoyait et au même moment Yves faisait la } \\
\text { vaisselle. }\end{array}$ \\
\hline Situations séquentielles & $\begin{array}{l}\text { Roger a fini son livre, et puis Charles est entré. } \\
\text { J’ai pris ma sacoche et puis je suis partie. }\end{array}$ \\
\hline $\begin{array}{l}\text { Situations sans spécification } \\
\text { explicite }\end{array}$ & $\begin{array}{l}\text { Eveline parlait à David, et Alexandre parlait à Nathalie. } \\
\text { Il est tombé sur ses genoux et il a commencé à pleurer. }\end{array}$ \\
\hline
\end{tabular}

Certains types de rapports sémantiques demandent quelques commentaires :

Les catégories causatif [1] et causatif [2] diffèrent dans ce sens que, dans le premier cas, une situation, généralement un événement ou une action, provoque directement une autre situation. Dans le second cas, par contre, le lien entre l'action ou l'événement 'causeur' et la situation 'causée' est moins direct. Cette distinction n'est cependant pas toujours facile à appliquer aux données concrètes que nous avons analysées.

Le nom de jussif est employé pour toute expression d'une demande, d'une exigence ou d'un commandement. L'attitude propositionnelle exprime l'attitude, le jugement ou l'opinion d'un participant vis-à-vis d'une situation donnée, tandis que la cognition 
représente plus abstraitement l'expression d'une connaissance générale ou d’une activité mentale.

La catégorie de la concession est censée réunir les adverbiales concessives traditionnelles, mais aussi les jonctions interpropositionnelles marquées par mais.

A partir de cette hiérarchie des rapports sémantiques entre les deux pôles, la RRG avance un certain nombre de prédictions dans le domaine de l'acquisition de ces types de liens, en particulier dans l'appropriation de la langue maternelle. Le développement langagier de la L1 chez les enfants dépend significativement de la structuration progressive du monde qui les entoure, entre autre par le biais de mots et de phrases. Notre étude vérifie si ces prédictions s'appliquent non seulement à l'acquisition d'une langue maternelle, mais aussi à celle d'une langue seconde ou étrangère.

\section{Méthodologie et questions de recherche}

Les données que nous avons analysées proviennent d’un projet plus vaste concernant la production orale en français et en anglais par des élèves de l'enseignement secondaire en Belgique néerlandophone, qui réalisent la même tâche narrative dans les deux langues. Ces deux langues constituent la première et la deuxième langue étrangère du programme scolaire en Flandre. La tâche consiste en une narration, basée sur une bande dessinée sans paroles, des aventures d'un petit garçon à la recherche de sa grenouille qui a disparu (la frog story ou “l'histoire de la grenouille”, Mayer 1969). Les apprenants ont dû produire la narration en temps réel, ce qui permet d’obtenir une production relativement spontanée en langue étrangère.

Les apprenants sélectionnés ont été répartis en quatre groupes, sur base de mesures globales liées à la correction de leur production orale. En particulier, nous avons pris en considération la proportion de propositions sans erreurs produites par les apprenants, l'adéquation de la conjugaison verbale, et l'emploi correct des prépositions dans la langue cible. Sur base de ces trois mesures, nous avons distingué quatre groupes, dont le premier représente le niveau de maîtrise le plus bas en français L2 et le quatrième le niveau le plus avancé (voir Buysse \& De Clercq (2014) pour un compte rendu détaillé de la constitution de notre corpus). A partir de là, nous avons analysé l'émergence des différents types des rapports sémantiques exprimés par la jonction interprédicationnelle en français dans la production de cinq apprenants par groupe. 
Les questions de recherche centrales à cette contribution sont basées sur les prédictions formulées par Van Valin (2001), qui s’appliquaient en premier lieu à l'acquisition d'une langue maternelle.

1. "In general, it is to be expected that children's first complex sentences would code the semantic relations at the top of the hierarchy, e.g. causality, mental dispositions such as desiring and wanting, and purpose” (Van Valin 2001: 14).

Sur le plan sémantique, les enfants acquérant leur L1 produiraient donc en premier lieu ces constructions qui servent le mieux leurs besoins immédiats, comme l'explicitation de leurs activités mentales (action psychologique, but, jussif) et des actions à conséquence directe (causatif). Il s’agit donc de catégories sémantiques qui se situent au sommet de la hiérarchie proposée par la RRG, et qui sont caractérisées par une relation étroite entre les deux événements liés, et d’ailleurs réalisées le plus souvent par le biais de connexions syntaxiquement sous-clausales. Sur le plan syntaxique d'ailleurs, les premières constructions complexes à surgir en acquisition d'une L1 selon la RRG sont des structures d'un niveau sous-clausal, c'est-à-dire nucléaire ou cellulaire, dans la mesure où les jonctions clausales impliquent plus d'actants et d'opérateurs grammaticaux et sont donc plus difficiles à produire.

Même à ce niveau sous-clausal, les types de rapports sémantiques qui se situent plus bas dans la hiérarchie (p. ex. la perception rapportée et les attitudes propositionnelles et cognitives) seraient moins saillants pour les jeunes enfants, et ne deviendraient donc fréquents ou même productifs à un stade plus tardif.

Nous allons examiner si ces prédictions s’appliquent non seulement aux jeunes enfants acquérant une première langue, mais également aux adolescents en train d'apprendre le français comme langue seconde ou étrangère. Nous formulons donc l'hypothèse que les relations sémantiques au sommet de la hiérarchie des rapports sémantiques (HRSmI) sont en effet productives avant celles qui se situent plus bas dans cette hiérarchie.

2. "Causative relations between events must involve a causer and a causee, and this entails that the actors of each nucleus in the juncture must be different. (...) samesubject constructions are less complex syntactically and therefore ought to appear before non-same subject ones” (Van Valin 2001:14). 
Van Valin (2001) souligne que les constructions causatives ne sont pas faciles à traiter et à produire en début d'acquisition, vu leur complexité structurelle ; elles requièrent l'union abstraite de deux événements, qui impliquent chacun des actants différents (exemples (1) et (2)). Par conséquent, elles sont peut-être produites moins spontanément que les liens d'action psychologique (exemple (3)) ou de but (exemple (4)), où le même actant est généralement impliqué dans les deux états d’affaires reliés.

(1) Je ferai nettoyer la cuisine à Benoît.

(2) Tu as laissé Christine manger les biscuits ?

(3) Guillaume essayera de finir ses devoirs aujourd'hui.

(4) Je fais du sport pour maigrir un peu.

Après avoir contrôlé si les relations sémantiques les plus étroites sont productives à partir des stades basiques, nous vérifierons l’hypothèse que le développement des jonctions à sens causatif est ralenti par rapport aux autres relations fortes entre prédications, à cause de leur complexité structurelle et conceptuelle plus marquée.

3. "Core junctures with transitive infinitival verbs will appear before nuclear junctures with transitive infinitival verbs, because core junctures present arguments in their canonical position with respect to their verbs while nuclear junctures do not” (Van Valin 2001:13).

Notre troisième question de recherche postule un lien entre l'acquisition d'une structure et la transparence des liens sémantiques au sein de celle-ci. Elle concerne les jonctions nucléaires et cellulaires, qui figurent dans la grande majorité des cas sous la forme de constructions transitives dans nos données. La fusion de deux prédicats en un seul prédicat complexe, exigée par la jonction nucléaire, entraîne une réorganisation de l'ensemble des arguments liés aux deux prédicats initiaux, ce qui rend ce type de construction assez difficile à envisager et à produire pour l'apprenant (exemple (1)). La jonction cellulaire, par contre, maintient généralement les arguments originaux dans leur position canonique (exemples (2) et (3)).

Dès lors, nous formulons la prédiction que les connexions au niveau cellulaire seront produites à un stade plus précoce que les nucléaires dans le développement des jonctions interprédicationnelles en L2 comme en L1. Les jonctions cellulaires, caractérisées par une connexion sémantique plus transparente entre les prédicats et leurs 
arguments, apparaîtraient donc avant les jonctions nucléaires, moins transparentes sur ce plan.

4. "In clausal junctures, the first semantic relations to be coded will be those at the bottom of the IRH” (Van Valin 2001:24).

Dans le cas des jonctions au niveau clausal, par contre, le développement des rapports sémantiques entre deux ou plusieurs unités se déroulerait de façon différente que pour les jonctions sous-clausales, du moins pour l'enfant acquérant sa langue maternelle. Le développement de la compétence narrative de l'enfant (Berman et Slobin 1994) serait caractérisé par l'apparition précoce des relations les moins étroites entre prédications selon la HRSmI, telles celle sans explicitation de rapport sémantique, ou marquant la simultanéité et la séquentialité. Les catégories plus élevées dans la hiérarchie n’apparaîtraient que plus tard dans la production de l'enfant.

La quatrième hypothèse formulée sera donc que les rapports sémantiques clausaux les plus lâches apparaîtront avant les plus intenses dans la production des apprenants d'une langue seconde ou étrangère également, comme ce serait le cas avec le développement de la compétence narrative en L1 chez les jeunes enfants.

\section{Données observées}

Dans les tableaux suivants, nous fournissons pour chacun des quatre groupes d'apprenants que nous avons distingués et pour un groupe de contrôle de dix locuteurs natifs du français le taux d'occurrence en pourcentage du total des liens sémantiques spécifiés par la HRSmI. Nous indiquons chaque fois par quel(s) type(s) de relation syntaxique la catégorie sémantique en question se trouve réalisée. ${ }^{1}$ Comme nos questions de recherche postulent des séquences de développement différentes selon le niveau syntaxique où la complexification a lieu, nous distinguerons les jonctions nucléaires et cellulaires d'une part des jonctions clausales d'autre part.

\footnotetext{
${ }^{1} \mathrm{Ncs}=$ cosubordination nucléaire ; Cecs = cosubordination cellulaire ; Cesc = subordination 'complément' cellulaire; Cesa $=$ subordination 'adverbiale' cellulaire ; $\mathrm{Ceco}=$ coordination cellulaire ; Clcs = cosubordination clausale ; Clsc = subordination 'complément' clausale ; Clsa = subordination 'adverbiale' clausale ; $\mathrm{Clco}=$ coordination clausale
} 


\subsection{Niveau 1}

\begin{tabular}{|l|l|l|l|l|l|l|l|l|l|}
\hline & Ncs & Cecs & Cesc & Cesa & Ceco & Clcs & Clsc & Clsa & Clco \\
\hline Causatif [1] & 1,3 & & & & & & & & \\
\hline Aspectuel & & & & & & & & & \\
\hline S-év. mod. & & 4,0 & & & & & & & \\
\hline Action psych. & & 1,3 & & & & & & & \\
\hline But & & & & & & & & & \\
\hline Jussif & & & & & & & & & \\
\hline Causatif [2] & & & & & & & & & \\
\hline Perc. directe & & & & & 1,3 & & & & \\
\hline Perc. indir. & & & & & & & 2,7 & & \\
\hline Att. propos. & & & & & & & & & \\
\hline Cognition & & & & & & & & & \\
\hline Discours ind. & & & & & & & 1,3 & & \\
\hline Discours dir. & & & & & & & 2,7 & & \\
\hline Circonstances & & & & 1,3 & & & & & \\
\hline Cause & & & & & & & & & \\
\hline Condition & & & & & & & & & \\
\hline Concession & & & & & & 1,3 & & & 16,0 \\
\hline Simultanées & & & & & & & & & \\
\hline Séquentielles & & & & & & & & & 2,7 \\
\hline Non explicites & & & & & & 13,3 & & & 50,7 \\
\hline
\end{tabular}

Au premier niveau de compétence, toutes les catégories sémantiques distinguées en RRG ne sont pas réalisées par les apprenants : des 22 catégories possibles, seules 13 présentent une ou plusieurs occurrences dans les productions des apprenants les moins avancés.

Aux niveaux syntaxiques sous-clausaux, c'est-à-dire nucléaire et cellulaire, quelques catégories en haut de la hiérarchie sémantique sont représentées, comme la causativité [1], le sous-événement modifiant et l'action psychologique. La perception directe et les circonstances spatio-temporelles, plus en bas dans la hiérarchie et censées donc réaliser un lien sémantique moins étroit entre les événements reliés, apparaissent également dans ces données. Le nombre de leurs occurrences est cependant très limité par rapport aux jonctions clausales, qui constituent la plus grande partie de l'ensemble des liens interprédicationnels dans ce groupe d'apprenants. 
Pour ce qui est du niveau clausal, certaines catégories placées au milieu de la hiérarchie sémantique sont présentes dans les données du premier groupe, notamment la perception indirecte et les discours indirect et direct. Les types de liens clairement majoritaires ici, toutefois, sont ceux qui se trouvent près du pôle "faible" de la hiérarchie : les relations temporelles et concessives ("mais”), et, surtout, les liens non spécifiés entre deux événements, qui représentent non moins de $64 \%$ de toutes les relations interprédicationnelles dans les productions des apprenants les moins avancés. Les apprenants construisent donc beaucoup de liens syntaxiques entre deux propositions entières, notamment de coordination et de cosubordination, sans expliciter la relation sémantique qui y correspond.

\subsection{Niveau 2}

\begin{tabular}{|l|l|l|l|l|l|l|l|l|l|}
\hline & Ncs & Cecs & Cesc & Cesa & Ceco & Clcs & Clsc & Clsa & Clco \\
\hline Causatif [1] & & & & & & & & & \\
\hline Aspectuel & & & & & & & & & \\
\hline S-év. mod. & & 9,2 & & & & & & & \\
\hline Action psych. & & & & & & & & & \\
\hline But & & & & & & & & 0,4 & \\
\hline Jussif & & & & & & & & & \\
\hline Causatif [2] & & & & & & & & & \\
\hline Perc. directe & & & & & & & 0,8 & & \\
\hline Perc. indir. & & & & & & & 0,8 & & \\
\hline Att. propos. & & & & & & & 1,2 & & \\
\hline Cognition & & & & & & & & & \\
\hline Discours ind. & & & & & & & 0,8 & & \\
\hline Discours dir. & & & & & & & & & \\
\hline Circonstances & & & & 2,0 & & & & & \\
\hline Cause & & & & & & & & 1,2 & \\
\hline Condition & & & & & & & & & \\
\hline Concession & & & & & & 0,4 & & & 6,8 \\
\hline Simultanées & & & & & & & & & \\
\hline Séquentielles & & & & & & & & & 1,2 \\
\hline Non explicites & & & & & & 6,4 & & & 68,7 \\
\hline
\end{tabular}


Le deuxième groupe d'apprenants produit très peu de liaisons interprédicationnelles aux niveaux sous-clausaux. Les cas de cosubordination cellulaire expriment tous le lien sémantique de sous-événement modifiant, situé vers le haut de la hiérarchie, et les quelques cas de subordination 'adverbiale' cellulaire produisent le sens circonstanciel spatio-temporel. Certaines catégories qui étaient représentées (bien que très rarement) dans les productions du premier groupe d'apprenants, comme la causativité [1] et l'action psychologique, sont absentes dans ce groupe, considéré pourtant comme plus avancé.

Les liaisons sémantiques interclausales, par contre, sont plus fréquentes et surtout plus variées qu'au sein du premier groupe d'apprenants. De nouvelles catégories sont représentées dans les narrations produites par le deuxième groupe, comme le but (assez en haut dans la hiérarchie, donc considérée comme un lien sémantique fort), l'attitude propositionnelle et la cause. A nouveau cependant, ce sont les relations les plus “faibles” qui représentent la partie majoritaire de toutes les jonctions réalisées à ce niveau ; les apprenants continuent clairement à produire des phrases complexes qui comportent bien une connexion syntaxique formelle, mais qui ne marquent pas explicitement la nature sémantique de la relation interclausale établie.

\subsection{Niveau 3}

\begin{tabular}{|l|l|l|l|l|l|l|l|l|l|}
\hline & Ncs & Cecs & Cesc & Cesa & Ceco & Clcs & Clsc & Clsa & Clco \\
\hline Causatif [1] & 2,8 & 0,3 & & & & & & & \\
\hline Aspectuel & & 0,3 & & & & & & & \\
\hline S-év. mod. & & 8,1 & & & & & & & \\
\hline Action psych. & & 0,3 & & & & & & & \\
\hline But & & & & & & & & 0,3 & \\
\hline Jussif & & & & & 0,7 & & 1,1 & & \\
\hline Causatif [2] & & & & & & & & & \\
\hline Perc. Directe & & & & & & & 2,1 & & \\
\hline Perc. indir. & & & & & & & 1,4 & & \\
\hline Att. propos. & & & & & & & 3,9 & & \\
\hline Cognition & & & & & & & 0,7 & & \\
\hline Discours ind. & & & & & & & 0,3 & & \\
\hline
\end{tabular}




\begin{tabular}{|l|l|l|l|l|l|l|l|l|l|}
\hline Discours dir. & & & & & & & & & \\
\hline Circonstances & & & & 4,6 & & & & & \\
\hline Cause & & & & & & & & 3,9 & \\
\hline Condition & & & & & & & & & \\
\hline Concession & & & & & & & & & 13,0 \\
\hline Simultanées & & & & & & & & & 0,7 \\
\hline Séquentielles & & & & & & & & & 6,3 \\
\hline Non explicites & & & & & & & & & 49,1 \\
\hline
\end{tabular}

Les relations sous-clausales sont mieux représentées dans les productions du troisième groupe que dans celles du deuxième. La causativité [1] est de retour, et les autres types de liens sémantiques "forts” vers le haut de la hiérarchie sont tous présents, même si leur nombre d'occurrences est souvent limité. La relation jussive apparaît pour la première fois dans les narrations des apprenants de ce groupe.

À part les types de liens situés près du pôle fort de la HRSmI, quasiment toutes les relations sémantiques possibles sont représentées dans les jonctions clausales des apprenants du troisième groupe. Il faut remarquer que la condition est absente, probablement pas en raison de son développement tardif dans le répertoire linguistique des apprenants, mais à cause de l'absence de contextes ou événements dans la narration originale nécessitant l'établissement de tels liens.

Même si la catégorie sans spécification explicite est toujours beaucoup plus importante que toutes les autres, elle perd en prépondérance, s'appliquant à 49,1\% des relations interprédicationnelles à ce stade, une décroissance nette par rapport au total de 75,1\% (cosubordination et coordination clausales) dans le groupe 2.

\subsection{Niveau 4}

\begin{tabular}{|l|l|l|l|l|l|l|l|l|l|}
\hline & Ncs & Cecs & Cesc & Cesa & Ceco & Clcs & Clsc & Clsa & Clco \\
\hline Causatif [1] & 3,1 & 0,7 & & & & & & & \\
\hline Aspectuel & & 1,7 & & & & & & & \\
\hline S-év. mod. & & 7,5 & & & & & & & \\
\hline Action psych. & & 3,1 & & & & & & & \\
\hline But & & & & & & & & 0,7 & \\
\hline
\end{tabular}




\begin{tabular}{|l|l|l|l|l|l|l|l|l|l|}
\hline Jussif & & & & & 1,4 & & 1,0 & & \\
\hline Causatif [2] & & & & & & & & & \\
\hline Perc. directe & & & & & & & 3,1 & & \\
\hline Perc. indir. & & & & & & & 2,4 & & \\
\hline Att. propos. & & & & & & & 0,7 & & \\
\hline Cognition & & & & & & & 1,0 & & \\
\hline Discours ind. & & & & & & & 0,3 & & \\
\hline Discours dir. & & & & & & & & & \\
\hline Circonstances & & & & 8,5 & & & & & \\
\hline Cause & & & & & & & & 3,8 & \\
\hline Condition & & & & & & & & & \\
\hline Concession & & & & & & & & & 6,8 \\
\hline Simultanées & & & & & & & & & 0,3 \\
\hline Séquentielles & & & & & & & & & 5,5 \\
\hline Non explicites & & & & & & 3,1 & & & 45,4 \\
\hline
\end{tabular}

Les apprenants du groupe le plus avancé produisent exactement les mêmes types de relations sémantiques aux niveaux nucléaire et cellulaire que les élèves du groupe 3 . Au niveau final, pourtant, la plupart de ces catégories (sauf la causativité [1] et le sousévénement modifiant) gagnent en importance par rapport à l'ensemble de liaisons interprédicationnelles produites. A part les circonstances spatio-temporelles, les liens réalisés se situent donc toujours vers le haut de la hiérarchie des relations sémantiques. Ce premier phénomène s’applique aussi aux jonctions clausales à ce stade : les catégories sémantiques qui connaissent des occurrences dans les productions du groupe 4 ne diffèrent pas de celles déjà présentes au niveau 3 , bien que leur proportion soit parfois légèrement différente. Le taux d’occurrence du type “mais” (considéré ici comme faisant partie de la catégorie concessive), notamment, perd la moitié de son importance en diminuant de $13,0 \%$ à $6,8 \%$ du troisième au quatrième niveau de compétence. A nouveau, donc, les liens sémantiques censés être les plus étroits sont les seuls à ne pas être représentés au niveau clausal dans les productions des apprenants. 
4.5 Type de production en français L2

Natifs:

\begin{tabular}{|l|l|l|l|l|l|l|l|l|l|}
\hline & Ncs & Cecs & Cesc & Cesa & Ceco & Clcs & Clsc & Clsa & Clco \\
\hline Causatif [1] & 10,2 & 0,3 & & & & & & & \\
\hline Aspectuel & & 3,2 & & & & & & & \\
\hline S-év. mod. & & 4,6 & & & & & & & \\
\hline Action psych. & & 2,5 & & & & & & & \\
\hline But & & & & & & & & 4,2 & \\
\hline Jussif & & & & & 2,1 & & & & \\
\hline Causatif [2] & & & & & & & & & \\
\hline Perc. directe & & & & & & & 0,7 & & \\
\hline Perc. indir. & & & & & & & 1,1 & & \\
\hline Att. propos. & & & & & & & 2,1 & & \\
\hline Cognition & & & & & & & 2,5 & & \\
\hline Discours ind. & & & & & & & 0,3 & & \\
\hline Discours dir. & & & & & & & 0,7 & & \\
\hline Circonstances & & & & 5,6 & & & & & \\
\hline Cause & & & & & & & & 7,0 & \\
\hline Condition & & & & & & & & & \\
\hline Concession & & & & & & 0,7 & & 0,3 & 4,9 \\
\hline Simultanées & & & & & & & & & \\
\hline Séquentielles & & & & & & & & & 6,3 \\
\hline Non explicites & & & & & & 4,6 & & & 36,1 \\
\hline
\end{tabular}

Les dix locuteurs natifs dont nous avons analysé les narrations produisent quasiment les mêmes types de liens sémantiques que les apprenants les plus avancés dans notre étude, à l'exception de la concession adverbiale (donc pas du type mais), qui n'est pas présente chez les apprenants de langue seconde, et qui d'ailleurs n'est pas requise par l'histoire de la grenouille ; ses occurrences font généralement partie de commentaires «metanarratifs», tels La forêt en elle-même, même s'il y a des choses terrifiantes, c'est toujours un superbe endroit (locuteur 742).

Les locuteurs natifs diffèrent bien des apprenants du groupe 4 en ce qui concerne la fréquence d'usage de certaines catégories sémantique. La causativité [1] est beaucoup 
mieux représentée chez les locuteurs de français L1 que chez les apprenants, entre autres à cause de l'emploi beaucoup plus fréquent de constructions comme Il s'est fait piquer par une abeille, où "fait piquer” constitue un seul prédicat complexe au niveau nucléaire. À l'autre pôle de l'échelle, les relations sémantiques du type situations sans rapport temporel explicite constituent toujours la catégorie la plus importante dans l'ensemble de relations sémantiques, mais sont employées moins souvent que dans les narrations de tous les apprenants, même les plus avancés. Ces derniers n’ont donc pas encore atteint une compétence quasi-native en ce qui concerne la liaison interprédicationnelle et interpropositionnelle, du moins d’un point de vue sémantique.

\section{Discussion des hypothèses formulées}

Dans ce qui suit, nous évaluons la validité des quatre hypothèses de recherche formulées dans la section 3.

1. Dans l'ensemble des relations sous-clausales (c'est-à-dire nucléaires et cellulaires), les liens sémantiques les plus forts se développent-ils avant les liens moins étroits?

Dès le premier niveau de compétence que nous avons étudié, certaines catégories sémantiques en haut de la HRSmI sont représentées dans les productions orales des apprenants. Il s'agit notamment de la causativité [1] - qui, d'ailleurs, est la seule forme de causativité présente dans le sous-corpus sous investigation - des sous-événements modifiants et des actions psychologiques. Dans l'ensemble de relations interprédicationnelles et interpropositionnelles réalisées dans ce premier groupe, ces catégories occupent bien une place mineure. D’autres types de liens sémantiques considérés comme moins étroits figurent aussi dans les narrations du premier groupe, comme le jussif (bien que très rare) et les circonstances spatio-temporelles. ${ }^{2}$

\footnotetext{
${ }^{2}$ La Grammaire du Rôle et de la Référence définit les subordonnées circonstancielles comme portant sur la cellule (le prédicat et ses arguments) et non sur la clause entière (qui comprend, outre à la cellule, des elements périphériques et potentiellement non obligatoires). Les unités syntaxiques réalisant ce type de lien sont donc analysées ici comme appartenant au niveau cellulaire, de puissance moyenne/ forte. Ceci semble contredire, pourtant, l'hypothèse générale de la RRG, qui prédit une correspondance maximale entre force syntaxique et sémantique des
} 
$\mathrm{Au}$ sein du deuxième groupe, les seules catégories sémantiques connaissant des occurrences au niveau sous-clausal sont les sous-événements modifiants et, de façon modeste, les circonstances spatio-temporelles ; en d'autres mots, les types de relations situés le plus haut dans la hiérarchie ne sont pas représentés à ce stade. Les apprenants du troisième groupe, cependant, emploient des connexions appartenant à toutes les catégories "fortes", même si ce sont toujours les sous-événements modifiants, les circonstances spatio-temporelles et cette fois-ci aussi la causativité [1] qui sont les plus importantes, les autres catégories n’étant représentées que très rarement. Ceci s’applique aussi largement au groupe 4, comprenant les élèves les plus compétents en français langue seconde dans cette étude. Dans ce dernier groupe, pourtant, les liens sémantiques moins étroits, comme le jussif et les circonstances spatio-temporelles, gagnent en importance.

En général, il semble correct que les liens sémantiques les plus forts sont réalisés par les apprenants à partir des stades les moins avancés de leur développement en français : la causativité [1], les sous-événements modifiants et les actions psychologiques sont déjà présents chez les locuteurs du groupe 1 , censés avoir une compétence très basique en français.

Trois types de relations sémantiques moins fortes sont également réalisés par le biais de structures syntaxiquement sous-clausales : le jussif (généralement réalisé par un cas de coordination cellulaire), la perception directe (subordination com-clausale ou, comme ici, coordination cellulaire) ${ }^{3}$ et les circonstances spatio-temporelles (subordination adcellulaire). Le jussif et les circonstances spatio-temporelles deviennent plus fréquents lorsque le niveau des apprenants progresse, ce qui concorde avec l'hypothèse formulée par Van Valin 2001.

La première hypothèse s'avère donc vraie dans une certaine mesure ; les relations sémantiques fortes (à part la causativité ; voir la deuxième question de recherche) semblent en effet être les plus importantes en début d'apprentissage, pour laisser ensuite

liens interprédicationnels, la catégorie sémantique circonstances spatio-temporelle se trouvant plutôt vers le pôle "faible" de la hiérarchie des relations sémantiques.

${ }^{3}$ Les cas de perception directe sont analysées différemment selon qu'elles sont formulées avec une clause entière à verbe fini (subordination ad-clausale ; J'ai vu que Louis pleurait) ou avec une cellule à verbe non fini (coordination cellulaire ; J'ai vu Louis pleurer). 
plus de place aux relations moins fortes comme le jussif et les circonstances spatiotemporelles. Il faut bien mentionner qu'il s'agit d'un changement de proportion et non d'une apparition soudaine de certaines structures à un moment donné ; quasiment toutes les catégories sémantiques examinées sont représentées dans les narrations des apprenants à partir du premier niveau de compétence.

2. Dans l'ensemble des relations les plus étroites entre prédications, le rapport causatif devient-il productif plus tardivement que les autres catégories situées du côté du pôle le plus fort ?

Comme mentionné précédemment, la causativité [1] est représentée dans les productions orales des apprenants à partir du niveau le plus basique. Elle ne surgit donc pas forcément plus tard dans le développement des apprenants de français L2, mais elle gagne bien en productivité lorsque les groupes progressent en compétence, allant d'un taux très bas de 1,3\% (groupe 1) à 2,8\% (groupe 3) et 3,1\% (groupe 4). (Elle est absente des narrations produites par les apprenants du deuxième groupe.) Ceci pourrait indiquer que les apprenants la trouvent en effet moins difficile à produire lorsqu'ils deviennent plus compétents en français L2. Les autres relations sémantiques fortes semblent donc perdre en importance avec le temps, pour laisser plus de place à la catégorie forte mais structurellement plus complexe de la causativité [1].

\section{Les jonctions cellulaires apparaissent-elles avant les jonctions nucléaires ?}

A partir du premier groupe, c'est-à-dire des apprenants à compétence très basique en français, des jonctions nucléaires ainsi que cellulaires sont réalisées, et ce constat s'applique toujours lorsque le niveau de français des élèves augmente, même si la catégorie syntaxique nucléaire est temporairement absente des narrations du groupe 2 . Les données ne confirment donc en tout cas pas l'idée que l'une apparaîtrait avant l'autre.

Nous pouvons effectivement observer que les jonctions nucléaires deviennent plus fréquentes lorsque la compétence des apprenants progresse : leur part du total croît de $1,3 \%$ à $2,8 \%$ et enfin à $3,1 \%$. Toutefois, la même tendance s’applique aux différentes catégories qui sont syntaxiquement productives au niveau cellulaire, à part les sousévénements modifiants, qui perdent légèrement en importance du premier groupe au 
quatrième. Le poids croisant de la jonction nucléaire n’est donc pas compensé par une décroissance de celui de la jonction cellulaire ou vice versa. L’hypothèse posée dans la section 2 n’est donc pas confirmée.

Soulignons encore que la variation sémantique au niveau de jonction nucléaire est très limitée, voire inexistante : le seul type de lien sémantique réalisé par le biais d’une liaison nucléaire est celui de causativité [1].

4. Dans l'ensemble des jonctions au niveau clausal, les relations au bas de la hiérarchie des relations sémantiques interprédicationnelles - c’est-a-dire les relations sémantiques considérées comme “faibles” - sont-elles acquises plus précocement que celles au milieu ou en haut de la hiérarchie ?

$\mathrm{Au}$ premier niveau de compétence, la grande majorité des liens au niveau clausal appartiennent aux catégories en bas de la hiérarchie des relations sémantiques. Les apprenants ne spécifient pas la nature sémantique de la connexion qu’ils établissent dans quasiment deux tiers des cas ; par conséquent, la classe sémantique des situations sans rapport temporel explicite est très bien représentée dans leurs narrations. Cette tendance est sans doute due en partie au fait que la tâche consiste en une narration orale quasispontanée durant laquelle les participants emploient très souvent la conjonction et comme moyen pour relier leurs phrases et maintenir la cohérence du discours ; en reliant des propositions entières, ils ne ressentent donc pas nécessairement le besoin d'indiquer une liaison sémantique particulière entre les deux situations ou événements en question.

La relation oppositive caractérisée par l'emploi du connecteur “mais”, classée au sein de la catégorie concessive, représente 17,3\% de tous les liens interprédicationnels à ce stade. La dernière catégorie “faible” à être représentée, bien que par très peu d'occurrences, est celle de séquentialité. Le milieu de la hiérarchie est également représenté dans les productions des élèves les moins compétents. À ce stade d'apprentissage du français, ils emploient occasionnellement des liens appartenant aux catégories perception indirecte, discours indirect et discours direct, ce qui n'est sans doute pas étonnant vu la saillance dans l'histoire de la grenouille des scènes où la protagoniste découvre que sa grenouille a disparu et où il s’adresse à son chien.

Le gamme sémantique de liaisons interprédicationnelles s’accroît progressivement dans les groupes suivants. Les élèves du deuxième groupe ajoutent à leur répertoire des cas de 
perception directe, d'attitude propositionnelle et de cause pour ce qui est des relations au milieu de la hiérarchie, donc des catégories où le lien n’est pas vraiment fortes ni clairement faible. Ils produisent aussi un nombre limité d'exemples exprimant le but, qui se trouvent plutôt du côté du pôle "fort” de la HRSmI. ${ }^{4}$ La prépondérance de la catégorie la plus “faible”, celle des situations sans rapport temporel explicite, s'accroît encore par rapport au niveau de compétence précédent. Dans les narrations du groupe 3 , les types de liens intermédiaires précédemment enregistrés sont produits plus fréquemment, et la variation dans l'ensemble des relations utilisées s'accroît : les sens de jussif (qui, d'ailleurs, peut être réalisé au niveau clausal tout comme au niveau cellulaire) et de cognition s’ajoutent à l'ensemble. Une répartition très semblable se maintient dans le groupe le plus avancé (groupe 4).

Les premières rapports sémantiques à apparaître en nombres considérables au niveau clausal sont en effet ceux qui se trouvent en bas de la hiérarchie sémantique et qui réalisent donc un lien assez faible entre les deux événements ou situations reliés. Après une poussée dans les productions du deuxième groupe, la catégorie des situations sans rapport temporel explicite perd en importance et cède du terrain aux alternatives avec explicitation du lien temporel ou avec “mais”. Les types de liens au milieu de la hiérarchie, qui ne sont donc considérés ni comme clairement forts ni comme clairement faibles, deviennent plus variés et connaissent plus de réalisations lorsque la compétence des apprenants s’accroît.

La quatrième hypothèse basée sur Van Valin 2001 est donc largement confirmée : les apprenants débutants produisent en effet plus de relations sémantiques “faibles” dans les jonctions au niveau clausal, et n’introduisent qu'à un niveau avancé de la variation dans la puissance des liens sémantiques qu'ils expriment, ce qui résulte en une décroissance de l’importance de la catégorie des événements sans ordre temporel.

\footnotetext{
${ }^{4}$ Mentionnons ici qu'un phénomène semblable mais inverse s'applique ici que nous avons observé pour la catégorie circonstances : la subordonnée de but est décrite en RRG comme s'appliquant syntaxiquement au niveau clausal au lieu de cellulaire. En d'autres mots, elle porte sur la proposition dans son entièreté, non seulement sur le prédicat et ses premiers arguments. Dans la HRSmI, pourtant, la catégorie de but se trouve entourée par des sens reflétées quasiment toujours par le biais de constructions cellulaires. Comme les circonstances, cette catégorie interrompt donc la correspondance supposément parfaite entre fortitude syntaxique et sémantique.
} 


\section{Conclusions}

Nous pouvons conclure de cet examen que les prédictions de la RRG sur le plan du développement des liens interprédicationnels sémantiques sont partiellement confirmées pour l'acquisition du français comme langue seconde.

Les deux premières hypothèse, concernant les jonctions aux niveaux nucléaire et cellulaire (donc sous-clausaux), sont largement confirmées par nos données. Comme prédit dans Van Valin 2001 pour l'acquisition de la langue maternelle, les relations sémantiques fortes, se trouvant en haut de la Hiérarchie des relations sémantiques interprédicationnelles, jouent un rôle important surtout chez les apprenants en début d'acquisition de la langue seconde. Même si d'autres types de liens, considérés moins étroits, figurent déjà dans les productions des apprenants les moins avancés, leur emploi est très limité au début et ne devient plus important que lorsque la compétence des apprenants progresse. Les liaisons sémantiquement fortes cèdent de la place à celles au milieu de la hiérarchie relationnelle. La causativité, censée être structurellement plus complexe que les autres relations sémantiques en haut de la hiérarchie, paraît en effet se développer plus tardivement chez les apprenants du français langue seconde tout comme chez ceux acquérant leur langue maternelle à un âge plus jeune. Les mêmes principes de complexité relative et de facilité d'acquisition semblent donc jouer pour ces deux catégories d'apprenants.

Notre hypothèse traitant de l'ordre de développement des jonctions au niveau clausal est également confirmée par les données analysées dans le cadre de cette étude. Les premières relations sémantiques produites en grands nombres par les apprenants du français langue seconde sont d'une nature sémantiquement implicite et appartiennent donc à la catégorie sans spécification explicite dans la hiérarchie ; ceci pourrait être dû en partie, chez les apprenants de notre étude tout comme chez les enfants parlant le français comme langue maternelle, à l'emploi très fréquent de et en tant que connecteur cohésif dans les discours oraux. La fréquence des autres relations en bas de l'échelle, comme les liens temporels et oppositifs (“mais”), n’est pas négligeable non plus, et ceci dès le niveau le moins avancé. Les types de liens d'une puissance moyenne, assez rares en début d'apprentissage, connaissent une plus grande variation et un nombre plus élevé d’occurrences lorsque la compétence des apprenants progresse. 
La quatrième hypothèse de recherche, de nature syntaxique mais basée également sur des principes sémantiques, n’a pas la mêmes pertinence pour les apprenants examinés ici que pour l'acquisition des langues maternelles (selon les prédictions de Van Valin 2001). Dans l'acquisition des liens interprédicationnels, les jonctions nucléaires n’apparaissent pas après les cellulaires, mais en même temps ; les deux catégories croissent en fréquence de manière similaire, en accord avec le développement de la compétence générale en L2. Ceci pourrait être dû au fait que la conceptualisation et la structuration mentale autour des prédicats (nucléaires) complexes seraient ce qui est difficile pour les jeunes enfants acquérant leur langue maternelle, et que ces principes sont déjà connus et maîtrisés par les apprenants du français comme langue seconde, qui peuvent s'appuyer dans ce domaine sur leurs propres compétences et connaissances en langue maternelle.

Sur le plan des relations sémantiques interprédicationnelles et interpropositionnelles, l'acquisition d'une langue seconde semble donc se dérouler de manière semblable à l'acquisition d'une langue première. Même si nous ne pouvons pas observer la totalité du parcours développemental depuis l'émergence jusqu’à la consolidation de la plupart des catégories que nous observons, un certain ordre de développement des rapports sémantiques exprimés est bien visible sous la forme d'un accroissement ou d'une diminution de la fréquence des catégories examinées. Ainsi, notre étude a permis de démontrer que la hiérarchie des relations interprédicationnelles sémantiques, proposée par la Grammaire du Rôle et de la Référence, génère des hypothèses de recherche fécondes pour rendre compte de l'évolution du répertoire de liens sémantiques exprimés selon l’âge et le niveau de compétence des apprenants.

\section{Bibliographie}

Benazzo, S. (2004). L’expression de la causalité dans le discours narratif en français L1 et L2. In : Langages, 155, pp. 33-50.

Buysse, M. (2014). Hiérarchie des relations syntaxiques interprédicationnelles et appropriation en français L2. In : Actes du CMLF 2014 - 4ème Congrès Mondial de Linguistique Française, EDP Sciences, 1419-1435, http://www.shsconferences.org/articles/shsconf/abs/2014/05/shsconf_cmlf14_01205/shsconf_cml f14_01205.html. 
Buysse, M. \& B. De Clercq. (2014). Matching the data: developing a multilingual corpus of language development. In : Née, E., Daube, J.-M., Valette, M., \& S. Fleury (éds.), Actes des 12es Journées internationales d'Analyse Statistique des Données Textuelles, 2014, pp. 529-538.

Degand, L. \& P. Hadermann. (2009). Structure narrative et connecteurs temporels en français langue seconde. In : Havu, E., Härmä, M., Helkkula, M., Larjavaara, M., \& U. Tuomarla (éds.), La langue en contexte. Actes du colloque "Représentations du sens linguistique IV”, Helsinki 28-30 mai 2008. Helsinki : Société Néophilologique, pp. 19-34.

Diessel, H. \& M. Tomasello. (2001). The acquisition of finite complement clauses in English: A corpus-based analysis. In : Cognitive Linguistics, 12, pp. 1-45.

Eckman, F., Moravcsik, E., \& J. Wirth. (1989). Implicational universals and interrogative structures in the interlanguage of ESL learners. In : Language Learning, 39, pp. 173-205.

Noyau, C. \& M.-T. Vasseur. (1986). L'acquisition des moyens de la référence temporelle en français langue étrangère chez des adultes hispanophones. In : Langages, 21 (84), pp. 105-117.

Van Valin, R. (2001). The acquisition of complex sentences: a case study in the role of theory in the study of language development. In : Boyle, J., Lee, J.-Y. \& A. Okrent (éds.), Chicago Linguistic Society, 36, pp. 14-39.

Van Valin, R. (2005). Exploring the syntax-semantics interface. Cambridge : Cambridge University Press.

Van Valin, R. \& R. J. LaPolla. (1997). Syntax: Structure, meaning and function. Cambridge : Cambridge University Press.

Reçu: 16 janvier 2015

Accepté: 11 juin 2015

Publié: 28 septembre 2015 